ReVISTA DE LA ESCUELA DE CIENCIAS DE LA EdUCACIÓN, AÑO 17, NRO. 16, VOL. 2, JULIO A DiCIEMBRE DE 2021. PÁGINAS 145-153. ISSN 2362-3349 (EN LíNEA). ESCUELA SECUNDARIA Y FORMACIÓN DOCENTE. SENTIDOS DE LA OBLIGATORIEDAD EN ESTUDIANTES DE PROFESORADO. LUCIANO DE MARCO.

\title{
ESCUELA SECUNDARIA Y FORMACIÓN DOCENTE SENTIDOS DE LA OBLIGATORIEDAD EN ESTUDIANTES DE PROFESORADO
}

\author{
Luciano De Marco* \\ Universidad de Buenos Aires, Argentina \\ luciano.demarco1@gmail.com
}

Recibido: 7/08/2020 - Aceptado: 1/12/2020

\begin{abstract}
Resumen
La masificación y obligatoriedad de la escuela secundaria —sancionada en el 2006 — replanteó los sentidos de un nivel signado por la selectividad desde su fundación. Esto convoca a la realización de reformas, modificaciones y variaciones en múltiples dimensiones y escalas para el sistema educativo incluyendo a la formación docente. A partir de una investigación exploratoria cualitativa de tipo inductiva finalizada, este artículo indaga cuáles son los sentidos sobre la obligatoriedad que expresan los/as estudiantes de dos profesorados -uno universitario y otro en un Instituto Superior de Formación Docente- para el nivel secundario ubicados en el Conurbano Bonaerense de la provincia de Buenos Aires. Desde un enfoque posestructuralista y con las herramientas conceptuales provistas por el materialismo cultural y el análisis político del discurso se desprende en las conclusiones que el sentido dominante de obligatoriedad se desarticula de los puntos nodales - como inclusión educativa o derecho a la educación- del discurso pedagógico estatal que primó hasta el 2015. Esto pone de manifiesto el carácter precario de la fijación de sentidos y específicamente una desarticulación entre los desafíos pedagógicos de las políticas educativas y las exigencias pedagógico-didácticas del trabajo de enseñar en las escuelas secundarias masificadas.
\end{abstract}

Palabras clave: Política educativa - Igualdad en educación - Formación docente - Escuela Secundaria - Obligatoriedad

\section{SECONDARY SCHOOL AND TEACHER TRAINING. TEACHER TRAINING STUDENT'S PERCEPTION MEANING OF COMPULSORY EDUCATION}

\footnotetext{
Abstract

The widespread growth and obligatory nature of secondary school — sanctioned in 2006rethought the senses of a level marked by selectivity since its foundation. This created challenges in multiple dimensions and scales for the educational system, including the teacher training. Based on a

* Profesor de nivel primario (ENS №4), licenciado y profesor en Ciencias de la educación (UBA) y magister en Ciencias sociales con orientación en educación (FLACSO). Actualmente es profesor en ISFD de la C.A.B.A. y becario doctoral (UBA) con sede en el IICE-UBA por el doctorado en educación en FFyL-UBA. Su área de investigación es la formación docente para el nivel secundario, en particular las políticas dirigidas hacia el sector y los vínculos entre los cambios culturales y los saberes de la formación.
} 
REVISTA DE LA ESCUELA DE CIENCIAS DE LA EdUCACIÓN, AÑo 17, NRO. 16, VOL. 2, JULIO A DiCIEMBRE DE 2021. PÁGINAS 145-153. ISSN 2362-3349 (EN LÍNEA). ESCUELA SECUNDARIA Y FORMACIÓN DOCENTE. SENTIDOS DE LA OBLIGATORIEDAD EN ESTUDIANTES DE PROFESORADO. LUCIANO DE MARCO.

completed qualitative inductive research, this article investigates the meanings of the compulsory nature expressed by the students of two teacher training - one university and the other in a Higher Institute of Teacher Training -for the secondary level located in the Conurbano Bonaerense of the province of Buenos Aires. From a poststructuralist approach and with the conceptual tools provided by the political analysis of discourse, in particular the concept of teaching positions, it follows in the conclusions that the dominant sense of obligation is disarticulated from the nodal points - such as educational inclusion or the right to education - of the State pedagogical discourse that prevailed until 2015. This highlights the precarious and not necessary character in the fixing of meanings and specifically a disarticulation between the pedagogical challenges of educational policies and the pedagogical-didactic demands of the work of teaching in current secondary schools.

Keywords: Teaching work - Educational equality - Teacher training - High school - obligatory nature.

\section{Introducción ${ }^{1}$}

La escuela secundaria se fundó a partir de un modelo institucional selectivo tanto en Argentina como en otros países de la región que moldeó la cultura escolar y las sensibilidades del trabajo de enseñar. En las últimas décadas, los sentidos en torno a este nivel escolar entraron en tensión debido a su masificación y al progresivo avance legislativo que culminó con la sanción de su obligatoriedad para toda la población en la Ley de Educación Nacional (LEN) en el 2006. Esto impactó en las políticas educativas en el nivel secundario orientadas hacia la construcción de una institucionalidad garante del derecho educativo entendido como el acceso, permanencia y egreso de la escolaridad; como la posibilidad de brindar contextos pedagógicos culturalmente ricos, diversos y democráticos y como un espacio de autorización cultural mediante experiencias igualitarias en el marco de una sociedad democrática.

Estos cambios en las agendas de las políticas educativas no son ajenos a la formación docente inicial (FDI), en particular al impulso de determinados saberes y temas emergentes que se engloban bajo la perspectiva de la educación como derecho: la educación sexual integral; los derechos humanos y las políticas de memoria; el trabajo pedagógico de reconocimiento de las heterogeneidades culturales; los andamiajes educativos para con los/as estudiantes provenientes de sectores vulnerabilizados para el logro de una igualdad de posiciones; la articulación de la enseñanza con la educación especial inclusiva; la incorporación en la enseñanza de artefactos digitales y medios conectivos con una mirada crítica sobre las posibilidades, potencialidades y límites que estos recursos dan.

Asimismo, estos cambios se inscriben en la formación docente junto con los propios que genera la reconfiguración del sistema formador en relación con su ampliación matricular y diversificación institucional (Birgin, 2017). La expansión matricular no impacta solamente en la escala del nivel, también supone la ampliación del origen socioeconómico-cultural de los/as estudiantes de profesorado. Se evidencia el ingreso a la educación superior de jóvenes provenientes de sectores sociales otrora excluidos, así como también del trabajo docente, y que son la primera generación que llega al nivel secundario y/o superior dentro de su familia (Birgin, 2015; Ezcurra 2011; Charovsky, 2013).

Por otra parte, la reconfiguración del sistema formador es acompañada por una variación institucional caracterizada por la creación del INFD en el 2007 y por el crecimiento y diversificación de instituciones que actualmente forman docentes. Este proceso de diversificación -iniciado en la segunda mitad del siglo XXtuvo tres olas expansivas en el ámbito universitario en las décadas de los '70, '90 y la primera década del siglo XXI (Chiroleu, Suasnábar y Rovelli, 2012) y estuvo acompañado por el crecimiento paulatino de los ISFD en las diferentes jurisdicciones del país. A partir del trabajo con diversas fuentes, Birgin plantea que entre 1970 y 2014 la cantidad de universidades creció un 245\% pasando de 29 a 100 y los ISFD crecieron un 929\% pasando de 215 a 2213 en el mismo período. Ahora bien, esta diversificación no se produce solo en términos cuantitativos sino también en la variedad de instituciones que forman docentes, incluyendo —por ejemploexperiencias de profesorados populares a cargo de organizaciones sociales en convenio con instituciones públicas (Birgin, Moscato e Ingratta, 2019; Birgin, 2017).

Inscribimos nuestros interrogantes en este escenario y bajo estos procesos de cambio. Como supuesto partimos de considerar que los modos en que se procesa la obligatoriedad involucran procesos complejos, paradojales y suponen diferentes dimensiones posibles de indagar. A partir de una mirada que piensa la formación docente desde una perspectiva pedagógica (Masschelein y Simons, 2014), recupera diversos estudios sobre las políticas educativas en torno a la escuela secundaria y utiliza herramientas conceptuales del materialismo cultural (Williams, 2009) y la teoría del discurso (Laclau y Mouffe, 1985), nos preguntamos:

\footnotetext{
${ }^{1}$ Este artículo es una revisión y ampliación de la ponencia presentada en el XIV Congreso Nacional de Ciencia Política "La política en incertidumbre. Reordenamientos globales, realineamientos domésticos y la cuestión de la transparencia" organizado por la Sociedad Argentina de Análisis Político y la Universidad Nacional de San Martín en julio de 2019.
} 
REVISTA DE LA ESCUELA DE CIENCIAS DE LA EdUCACIÓN, AÑo 17, NRO. 16, VOL. 2, JULIO A DiCIEMBRE DE 2021. PÁGINAS 145-153. ISSN 2362-3349 (EN LÍNEA). ESCUELA SECUNDARIA Y FORMACIÓN DOCENTE. SENTIDOS DE LA OBLIGATORIEDAD EN ESTUDIANTES DE PROFESORADO. LUCIANO DE MARCO.

¿Cuáles son los sentidos en torno a la obligatoriedad de la escuela secundaria de los/as estudiantes de profesorado ${ }^{2}$ ?

Recuperamos los resultados parciales de una investigación finalizada que produjo una tesis de maestría metodológicamente inscripta en el paradigma interpretativo y con un diseño de investigación cualitativo de tipo inductivo. Se trata de un estudio con un carácter exploratorio sin pretensiones de generalización. La muestra seleccionada proviene de dos profesorados, uno universitario y otro en un Instituto Superior de Formación Docente (ISFD) en áreas de conocimiento afines a las Ciencias Sociales. La elección de las instituciones estuvo motivada por la vacancia de investigaciones sobre formación docente en la región del Conurbano Bonaerense en la Provincia de Buenos Aires y en el ámbito universitario en general. Entre 2016 y 2017 se realizaron entrevistas en profundidad a directivos/as, coordinadores/as, profesores/as de la residencia docente y estudiantes que estuviesen cursando esta asignatura. La información obtenida fue leída en una trama de sedimentaciones e hibridaciones en la que se anclan los sujetos entrevistados y en las relaciones que estos establecen con los discursos que circulan en la sociedad.

Para desarrollar nuestro argumento, en el primer apartado presentamos brevemente el marco teórico desde el que reflexionamos acerca de la construcción de sentidos y la lucha por estos. En el siguiente apartado, recuperamos estudios sobre el vínculo entre escuela secundaria y obligatoriedad para reflexionar sobre los desafíos que ese vínculo genera en la formación docente. En el tercer apartado presentamos los resultados obtenidos en la investigación para dar una respuesta tentativa a la pregunta anteriormente formulada. Finalizamos con algunas reflexiones sobre los modos de procesar la obligatoriedad en estos profesorados y con algunos interrogantes para futuras investigaciones.

\section{Notas conceptuales acerca de los sentidos en el ámbito educativo}

Para abordar nuestra pregunta por los sentidos en torno a la obligatoriedad de la escuela secundaria en la FDI partimos de una perspectiva posestructural que intenta articular elementos del materialismo cultural de Raymond Williams (2009) con los aportes de la teoría del discurso de Ernesto Laclau y Chantal Mouffe (2015).

Los aportes de Williams (2009) nos permitirán pensar los modos en que los sentidos en torno a la obligatoriedad se inscriben en la tradición pedagógica de la escuela secundaria. Es decir, como una selección y reselección de elementos significativos del pasado, recibidos y recuperados, que representan no una continuidad necesaria, sino deseada en el presente. Como toda tradición, está compuesta por elementos residuales, dominantes y emergentes. Respecto de los residuales, si bien se configuraron en un tiempo pasado mantienen su vigencia y significatividad en la actualidad. Los elementos dominantes se caracterizan por su eficacia en el grado de integración y circulación que presentan entre las tradiciones y las instituciones. Finalmente, los elementos emergentes refieren a nuevos significados y valores, a las nuevas prácticas, las nuevas relaciones y tipos de relaciones creadas. Al respecto, intentaremos identificar elementos dominantes, residuales y emergentes en las formas de comprender lo obligatorio de la escuela secundaria.

Por otra parte, desde la perspectiva de Laclau y Mouffe (2015), lo existente guarda estrecha vinculación con la significación construida a partir de una práctica articulatoria entendida como toda práctica que establece una relación tal entre los elementos que, a partir de la práctica, la identidad de estos resulta modificada. A partir de la totalidad estructurada resultante de esa práctica articulatoria se conforma un discurso. En ese sentido, la misma posibilidad de percepción, pensamiento y acción depende de la estructuración de un cierto campo significante que preexiste a cualquier inmediatez factual. Estos rasgos de la totalidad discursiva plantean la imposibilidad de fijación última de sentido, en tanto que solamente puede haber fijaciones parciales. De lo contrario, no sería posible dar lugar a las diferencias o a la subversión en los sentidos que se le otorgan a la realidad social. Incluso, para diferir o para subvertir un sentido tiene que haber uno fijado ante el cual diferenciarse. En ese sentido, Laclau y Mouffe (1985) plantean que el discurso se constituye como intento de dominar el campo de la discursividad, pese a la precariedad de toda fijación y para esto es preciso constituir un centro denominado punto nodal que es ocupado por los significantes privilegiados que fijan el sentido de la cadena significante.

Los aportes de Laclau y Mouffe declinan en el campo educativo en la categoría de posiciones docentes (Southwell,2008; Vassiliades, 2012). Desde este concepto los problemas educativos que otorgan sentido al trabajo de enseñar no son fijos sino abiertos, contingentes y construidos por una multiplicidad de factores. Estos devienen del vínculo complejo entre diferentes discursos, construidos social e históricamente acerca de diferentes problemas educacionales que se encuentran sedimentados y rearticulados en el presente. Los sujetos se sirven de diferentes discursos sobre lo educativo para dar forma y dotar de sentido a su práctica y definir los problemas, aspiraciones y utopías que el trabajo de enseñar involucra mediante una negociación siempre densa y compleja entre los discursos disponibles y los contextos en los que los sujetos se inscriben (Southwell, 2008; Vassiliades, 2012). Las posiciones docentes configuran formas de sensibilidad y de interpretación acerca del proceso de aprendizaje de los aprendices, del trabajo y las exigencias propias de los enseñantes. En virtud de esto, los sentidos sobre lo escolar y los saberes que los sujetos entienden

2 Denominamos "profesorado" a las carreras de educación superior que forman docentes independientemente de que funcionen en Universidades o Institutos Superiores de Formación Docente. 
REVISTA DE LA ESCUELA DE CIENCIAS DE LA EdUCACIÓN, AÑo 17, NRO. 16, VOL. 2, JULIO A DiCIEMBRE DE 2021. PÁGINAS 145-153. ISSN 2362-3349 (EN LÍNEA). ESCUELA SECUNDARIA Y FORMACIÓN DOCENTE. SENTIDOS DE LA OBLIGATORIEDAD EN ESTUDIANTES DE PROFESORADO. LUCIANO DE MARCO.

como necesarios para el trabajo docente son fluctuantes y se encuentran en disputa. Estos sentidos y saberes se encuentran entrelazados con aquello que los sujetos definen como lo justo, lo bueno, lo deseable y lo rechazable en las situaciones escolares cotidianas (Southwell, 2013; Vassiliades, 2012).

A partir de estos marcos conceptuales surge la pregunta sobre los sentidos en torno a lo obligatorio de la escuela secundaria en la formación de docentes para este nivel educativo. A modo de hipótesis sospechamos que en los modos de comprender cómo era la escuela secundaria en el pasado y qué significa su obligatoriedad en el presente se juega una mirada sobre cuáles son los problemas educativos y una sensibilidad particular por atenderlos.

\section{Escuela secundaria y obligatoriedad. Interpelaciones a la formación inicial de sus docentes}

La escuela secundaria en Argentina se configuró a partir de un modelo institucional tradicional que generó condiciones para la expansión-incorporación constantemente de matrícula como también para la expulsión y el desgranamiento (Acosta, 2012). Esta se fundó bajo una matriz selectiva que forjó una forma escolar (Vincent, Lahire y Thin, 2001) con un saber organizado en disciplinas; un currículum generalista, enciclopédico y graduado, con una enseñanza en la que la simultaneidad y la exposición magistral fueron piezas clave ante un estudiantado agrupado según su edad en aulas o secciones (Southwell, 2011).

Ahora bien, la selectividad comenzó a erosionarse a medida que el nivel educativo empezó a masificarse en una larga marcha iniciada a mediados del siglo XX. En lo que se refiere a las últimas décadas, mientras que la población en edad escolar (13 a 17 años) que asistía a la escuela secundaria era del 42,2\% en 1980 , en 2006 era del $86 \%$ y en el 2011 del $92,9 \%$. Además, en la primera década de este siglo crecieron las tasas netas de escolarización pasando del 81,4\% en 2000 al 84,5\% en 2010. Por último, la diferencia entre los que ingresan y los que la finalizan también mejoró al mostrar un decrecimiento del 37,8 al 32,8 (Itzcovich, 2014 en Southwell, 2018). Esta impugnación de la selectividad también tuvo paulatinos avances legislativos como fue la obligatoriedad del tercer ciclo con la Ley Federal de Educación (1993) o del nivel secundario en algunas jurisdicciones como en la Ciudad Autónoma de Buenos Aires (2002). En esta impugnación a la selectividad se inscribe la obligatoriedad del nivel secundario para toda la ciudadanía sancionada por la LEN. De este modo, la extensión del derecho a la educación se traduce en el derecho a la escolarización en sintonía con el debate y reformas que hubo en la región de América Latina entre fines del siglo XX y comienzos del XXI (Acosta, 2020). Ese derecho a la escolarización supone analizar la vida cotidiana de las escuelas para intentar impugnar, modificar, alterar las inercias que persisten por una selectividad en el nivel secundario.

Al respecto, hay una amplia literatura académica sobre las dimensiones que la obligatoriedad convoca a repensar, modificar, suprimir y crear. Cuestiones como los regímenes académicos, los modelos institucionales, los modos de fundar la autoridad pedagógica, el lugar otorgado a la participación estudiantil y los modos de enseñar y evaluar son algunas de las dimensiones que son necesarias discutir para que el derecho a la educación se haga lugar en la escuela secundaria. Es decir, la obligatoriedad supone nuevas exigencias para las instituciones (y para quienes allí trabajan de enseñar) que deben establecer mayores diálogos con la heterogeneidad de realidades, territorios y contextos en los que las escuelas secundarias se despliegan como también con la diversidad de sujetos que ingresan al nivel con trayectorias educativas que distan en muchos casos de las teóricas. Por lo tanto, la obligatoriedad del nivel secundario interpela a la política educativa - pese a la interrupción y retroceso que supuso la agenda gubernamental en educación de los últimos años - para promover cambios normativos, institucionales, curriculares y del régimen académico en diferentes escalas y niveles de implementación para dar lugar al derecho que esta anuncia (Capellacci y Miranda, 2007; Terigi, 2008; Giovine y Martignoni, 2011; Martignoni, 2012; Más Rocha, Gorostiaga, Tello y Pini, 2012; Montes y Ziegler, 2012; Acosta, 2012; Krichesky, 2013; Schoo, 2013; Poggi, 2018 ; Dussel, 2015 ; Pinkasz, 2015).

Estas variaciones y resignificaciones de la escuela secundaria producto de su obligatoriedad y masificación interpelan también a la formación inicial de sus profesores/as. Además de dar lugar a temas y saberes emergentes en la formación docente como los descriptos en la introducción, generan otros también susceptibles de ser abordados en los diferentes campos de la FDI como son: la preparación para ejercer la docencia utilizando otras estrategias didácticas y distintos dispositivos pedagógico-didácticos para enseñar (Falconi, 2016) en el marco del declive de la clase expositiva; la formación para establecer relaciones con los saberes más exhaustivas e igualitarias en tiempos acelerados por la cultura digital y visual (Dussel, 2015) y en donde las formas atencionales dominantes distan de las escolares (Larrosa, 2019); la construcción de una autoridad pedagógica democrática (Greco, 2007) que habilite un espacio en común que habitar y por último conocer en los espacios curriculares de práctica docente territorios y contextos educativos diferentes a los transitados durante las trayectorias educativas previas para resignificar las aspiraciones laborales y empatizar con las diversas realidades en las que las escuelas secundarias funcionan.

Nuestra pregunta, que a continuación profundizaremos, pretende dimensionar otras exigencias que interpelan a la FDI en clave de construir posiciones docentes sensibles ante los desafíos pedagógicos que la obligatoriedad escolar anuncia. 
ReVISTA DE LA ESCUELA DE CIENCIAS DE LA EdUCACIÓN, AÑo 17, NRO. 16, VOL. 2, JULIO A DiCIEMBRE DE 2021. PÁGINAS 145-153. ISSN 2362-3349 (EN LíNEA). ESCUELA SECUNDARIA Y FORMACIÓN DOCENTE. SENTIDOS DE LA OBLIGATORIEDAD EN ESTUDIANTES DE PROFESORADO. LUCIANO DE MARCO.

\section{Los sentidos en torno a la obligatoriedad de la escuela secundaria}

La escuela secundaria se construyó en la tensión entre selectividad y masificación/obligatoriedad. Por ello recuperaremos en primer lugar los sentidos en torno a la selectividad para luego adentrarnos en la obligatoriedad.

\section{“La escuela de antes" en tiempo presente. La persistencia de la selectividad}

Proponemos concebir la selectividad y la obligatoriedad como significantes que expresan discursos antagónicos con prácticas articulatorias disimiles (Laclau y Mouffe, 2015) que encadenan significantes específicos. La selectividad es un fenómeno social de diferenciación y construcción de elite que supone una escuela para pocos/as en función de la herencia recibida o el mérito demostrado. Contrariamente, la obligatoriedad expresa la pretensión de "universalizar" el nivel ya que implica efectivizar un derecho en vez de usufructuar un privilegio.

A partir del sentido que hagan estos discursos en los sujetos se forman sensibilidades particulares que enuncian problemas educativos específicos y suponen formas de ejercer el trabajo de enseñar diferentes. Según qué discurso tenga mayor fijación en los sujetos, las coordenadas bajo las que se interpretan cuestiones como las situaciones de desgranamiento o repitencia; los problemas en el aprendizaje; el lugar de la participación estudiantil en la institución, las formas de abordar la convivencia escolar serían distintas.

Teniendo como referencia la selectividad como construcción discursiva analizaremos los relatos de los/as estudiantes entrevistados/as sobre "la escuela de antes". En estos relatos, los/as estudiantes representan dicha escuela a partir de su experiencia en el nivel secundario, en las escuelas asociadas ${ }^{3}$ y de la formación académica que recibieron. Algunos estudiantes dijeron lo siguiente:

¿Vos no aprendiste? Te vas. ¿No cumpliste con los requisitos? te vas. Tengo que empezar a pensar cómo modifico el plan de estudios. No es justo que para un pibe que está en segundo año, que rinde bien todas las materias menos tres se tenga que ir (como hacen en las escuelas privadas) o volvés a hacer todo de vuelta. Entonces si cambiamos la lógica capaz que podría ser un modelo más ideal (E1 -31 años - Escuela secundaria pública - Universidad - Comunicación Social) ${ }^{4}$.

Con "la escuela no es lo que era antes" están haciendo alusión a la vieja escuela, sarmientina, disciplinadora, tradicional. La escuela homogeneizadora que tiene que responder a un proyecto de Nación, de país. Y ahora todos los valores están trastocados, hay como un corrimiento de todo eso y hay mucha heterogeneidad de pensamiento, de valores, creencias, la cultura, la socialización y la acción mediática lleva a un ejercicio diario muy fuerte en las personas (E2 - 61 años - CENS - Universidad - Comunicación social).

Lo que era "antes" ... era un grupo de gente que estaba ahí para escuchar al profesor, tomando una distancia determinada, que no volaba una mosca y el profesor era el que decía o hacía, no había casi participación y solo eran preguntas y respuestas o respuestas de cuestionarios escritos. Una escuela muy dirigida desde un púlpito donde no podías abrir la boca (E3 - 50 años - Escuela secundaria pública - ISFD - Geografía).

La "escuela de antes" como representación se vincula con el sentido de la selectividad como un sentido residual (Williams, 2009) ya que si bien fue formado en el pasado es un elemento efectivo del presente. Al respecto, la selectividad opera como un sentido sedimentado de la escuela secundaria en el que los vínculos entre docentes y alumnos/as son más formales, distantes y asimétricos, los/as estudiantes deben adaptarse al contexto escolar ante la posibilidad de ser excluidos y es una sensibilidad en donde conocer las identidades, culturas y saberes que portan los/as alumnos/as no es de relevancia.

\section{¿A quién le compete la obligación? Tensiones en torno al sentido de la obligatoriedad}

La forma de comprender la obligatoriedad de la escuela secundaria se enhebra con procesos previos como la obligatoriedad del nivel primario y la selectividad de la escuela secundaria. Para eso, el concepto de tradición (Williams,2009) -entendido como un proceso siempre selectivo de un pasado configurativo y de un presente preconfigurado - es fecundo para comprender la trama en la que la obligatoriedad de la escuela secundaria se inscribe. Una tradición compuesta por elementos dominantes y otros emergentes en pugna. En las entrevistas realizadas, los sentidos en torno a la obligatoriedad de la escuela secundaria que surgieron fueron los siguientes:

Pensaron en meter a todos los pibes a la escuela secundaria y que sea obligatoria porque todos se lo merecen. Pero pasa que cuando metemos diez y uno no quiere, no sé si ahí lo estamos incluyendo u obligando (E4 -44 años - Escuela secundaria pública - ISFD- Historia).

\footnotetext{
${ }^{3}$ Denominamos como "escuelas asociadas" a las escuelas secundarias en donde los/as estudiantes realizan las prácticas docentes del Campo de la Formación para la Práctica Profesional.

${ }^{4}$ En las citas de los/as entrevistados/as se incorporó la siguiente información con el objetivo de contextualizar sus relatos: edad, tipo de gestión de la escuela secundaria en la que estudió, institución y carrera en la que estudia el profesorado. A su vez se tomó la decisión de no rectificar las construcciones gramaticales de los testimonios.
} 
REVISTA DE LA ESCUELA DE CIENCIAS DE LA EdUCACIÓN, AÑo 17, NRO. 16, VOL. 2, JULIO A DiCIEMBRE DE 2021. PÁGINAS 145-153. ISSN 2362-3349 (EN LÍNEA). ESCUELA SECUNDARIA Y FORMACIÓN DOCENTE. SENTIDOS DE LA OBLIGATORIEDAD EN ESTUDIANTES DE PROFESORADO. LUCIANO DE MARCO.

La palabra obligatoriedad no me gusta, pero es lo que hay. Porque en realidad tendría que ser una opción, todo el mundo debiera ir, pero no por obligación (E3 - 50 años - Escuela secundaria pública ISFD - Geografía).

Se confunde obligatorio con necesario. A los pibes les decís que es obligatorio y es como una imposición. Habría que mostrarles la necesidad de esa escuela, de que tienen que ir a la escuela... La obligatoriedad hay que romperla para mostrar la necesidad detrás de la obligación (E8 - 33 años Escuela secundaria privada - ISFD - Historia).

No me gusta la palabra obligatorio porque es como que a uno lo llevan forzadamente, por la connotación, pero con la posibilidad que todos los jóvenes y adolescentes puedan estudiar en ese nivel (E2 - 61 años - CENS - Universidad - Comunicación social).

Creo que es comprender que la educación es un derecho. Van [los/as estudiantes] como diciendo "Me obligan a venir acá". Entonces cambiar la visión: "Me obligan a pensar que es un derecho". "No pensarla desde una obligación sino pensarla desde un lugar de derecho (E1 - 31 años - Escuela secundaria pública - Universidad - Comunicación Social).

Estos testimonios y otros similares asignan la responsabilidad de asistir a la escuela en los/as alumnos, independientemente de su voluntad y de las condiciones materiales y simbólicas que lo habilitan. Pareciera que la obligatoriedad no permea a las condiciones institucionales para recibir a los alumnos/as. De algún modo, en estas formulaciones se actualizan las articulaciones que comprenden los problemas educativos como asuntos individuales a la vez que diluyen los espacios de construcción de comunidad, de un lazo colectivo, de un espacio en común (Vassiliades, 2020). Este sentido de obligatoriedad se vincularía con la forma decimonónica de articular el trabajo docente con la igualdad educativa (Vassiliades, 2015); opera la lógica de la imposición de derechos (Sarlo, 1998) que a la vez que impone una identidad en los sujetos que se inscriben en lo escolar, los habilita a mejores condiciones de existencia. También se articularia con una noción de igualdad en donde la anulación de las diferencias o de inclusión por homogeneización (Dussel, 2004) traduce la igualdad en ser "lo mismo" dada la condición de amenaza o deficiencia de las diferencias a la vez que supone resignar, renunciar, dejar de lado parte de la propia identidad como condición para incluirse.

Por lo tanto, la obligatoriedad no se articula con la creación de un espacio educativo de posibilidad, de realización, de crecimiento, sino como una condición de imposición en la que los sujetos "algo" tienen que ceder. Al respecto, no se recupera el sentido político que detenta la obligatoriedad y su pluralidad de significaciones, tampoco una historización de esta en el sistema educativo y una reflexión sobre cómo se vinculan los derechos de los/as ciudadanos/as con las responsabilidades estatales en sus diversas escalas de concreción.

Ahora bien, recuperaremos otros testimonios que abonarían otro sentido sobre la obligatoriedad. En las entrevistas realizadas, algunos/as estudiantes planteaban lo siguiente:

Si es obligatoria, debería haber políticas que garanticen que sea obligatoria y entonces es un paso más a que sea de calidad. El mayor desafío es lograr que los chicos del secundario sigan estudiando y romper con esta reproducción de clases. El desafío está desde las políticas, pero también desde el hacer cotidiano. Si el profesor incentivo a los chicos a que pueden, a que son capaces de estudiar, que haya becas (E7 - 25 años - Escuela secundaria pública - Universidad - Comunicación social).

No deja de ser una ley necesaria, pero necesita ser acompañada por una cantidad de cosas que la hagan efectiva. ¿Va a haber que cambiar estrategias? Quizás sí, ¿habrá que hacer las cosas de otra manera? Probablemente sí. ¿Van a tener que tomarse otros tiempos? Si ¿Va a tener que cambiar el currículum? Probablemente ¿Los docentes van a tener que trabajar distinto y pensar de otra manera? Probablemente (E8 - 27 años Escuela secundaria privada - Universidad - Comunicación social).

En estos relatos, la obligatoriedad se encadena con las responsabilidades del Estado, las instituciones y/o la docencia por producir condiciones sociales, culturales, pedagógicas y didácticas que hagan posible la escolarización de la ciudadanía y la apropiación de los saberes por parte de los/as estudiantes. A su vez, estos relatos encadenan la obligatoriedad con una hipótesis de igualdad de inteligencias (Rancière, 1987) de quienes asisten a la escuela secundaria ("que los chicos pueden"). Esta noción que articula docencia con igualdad nos refiere al potencial que tienen los discursos para fijar sentido en los sujetos, siempre mediante procesos sumamente complejos y no lineales. Especialmente el discurso que ligó en las últimas décadas lo educativo con la igualdad y con lo común y que tuvo momentos de gran capacidad de fijación al punto tal que puede encontrarse plasmado en la Ley de Educación Nacional. Por ejemplo, en el artículo 71 define la identidad docente en los siguientes términos: Promoverá la construcción de una identidad docente basada en la autonomía profesional, el vínculo con la cultura y la sociedad contemporánea, el trabajo en equipo, el compromiso con la igualdad y la confianza en las posibilidades de aprendizaje de los/as alumnos/as.

Estamos muy distantes de considerar esta relación como un proceso de implementación "eficaz" de la política educativa. A partir de la producción conceptual de Ball (2015), es conocida la complejidad que 
REVISTA DE LA ESCUELA DE CIENCIAS DE LA EdUCACIÓN, AÑo 17, NRO. 16, VOL. 2, JULIO A DiCIEMBRE DE 2021. PÁGINAS 145-153. ISSN 2362-3349 (EN LÍNEA). ESCUELA SECUNDARIA Y FORMACIÓN DOCENTE. SENTIDOS DE LA OBLIGATORIEDAD EN ESTUDIANTES DE PROFESORADO. LUCIANO DE MARCO.

supone la política educativa como puesta en acto y los diversos modos de traducción en las diferentes escalas del sistema educativo. Más bien, la relación anteriormente marcada nos resulta pertinente para ilustrar la dispersión que caracteriza a los discursos y de las formas imbricadas en que se construyen los encadenamientos a partir de los que los sujetos le otorgan sentido a la realidad educativa como a su propia tarea docente.

Como acabamos de ver la obligatoriedad de la escuela secundaria tiene, al menos, dos encadenamientos diferentes que pugnan por el sentido de esta. En uno se la encadena con la responsabilidad del individuo por su proceso educativo y con la noción de imposición. Este encadenamiento podría vincularse con la forma sedimentada de comprender la obligatoriedad de la escuela primaria desde sus orígenes y por lo tanto creemos que es un sentido dominante. En cambio, en el segundo la obligatoriedad se encadena con una responsabilidad que cae en la capacidad que tengan las instituciones de construir condiciones propicias para la escolarización y la apropiación de los saberes y en la hipótesis de igualdad con respecto a las posibilidades de los/as alumnos/as del nivel. Este sentido se articula con el discurso emanado desde las políticas educativas de la primera década y media del siglo XXI que se organizó a partir de dos cadenas de significación. Una que articuló el derecho a la educación, el rol principal del Estado en educación y una noción de igualad vinculada con lo común y la diversidad junto con otra cadena que buscó articular igualdad e inclusión poniendo como centro a la enseñanza, el trabajo en situaciones de desigualdad y la alteración de los modos de organización escolar y de los regímenes académicos (Vassiliades, 2015, 2020). Debido a ello creemos que puede considerarse un emergente en la tradición pedagógica de la escuela secundaria argentina.

Por último, a los/as estudiantes les preguntamos qué cuestiones de la formación recibida se relacionaban con enseñar en una escuela secundaria recientemente obligatoria. Al respecto encontramos escasas respuestas. Se esperaba encontrar algunas reflexiones que articulen los desafíos que la política educativa generó a partir de la sanción de la obligatoriedad con las exigencias que esta supone para la tarea docente. Estamos lejos de entender esta articulación como necesaria ya que se aleja de una mirada inductiva; más bien creemos que esto refiere al vínculo endeble, no siempre explicitado, entre política educativa y exigencias pedagógico-didácticas para ejercer el trabajo de enseñar.

\section{Reflexiones finales}

Este artículo intenta aportar elementos para una pregunta más amplia sobre cómo construir durante la FDI posiciones docentes sensibles a los desafíos pedagógicos que anuncia la obligatoriedad escolar y que declinan en una pregunta por la igualdad como punto de partida (Rancière, 1987). Iniciamos este escrito con la siguiente pregunta: ¿cuáles son los sentidos que construyen los/as estudiantes de profesorado en torno a la obligatoriedad de la escuela secundaria? Describimos dos encadenamientos disímiles que no agotan la diversidad de otros encadenamientos posibles. Uno dominante que encadena la responsabilidad de la escolarización al alumno/a y su entorno próximo y se vincula con el sentido que adoptó la obligatoriedad del nivel primario a fines del siglo XIX en que la noción de igualad se asoció con "lo mismo". En cambio, el otro sentido - que comprendemos como emergente, con avances y especialmente retrocesos en los últimos años - responsabiliza al Estado, las instituciones y sus docentes de la escolarización en la escuela secundaria y de los aprendizajes que los/as alumnos/as allí realicen. Este último sentido se asocia con el discurso que acompañó las políticas educativas emanadas por el Estado Nacional durante la primera década y media del siglo XXI y que asoció la noción de igualdad con lo común.

En esta línea, encontramos evidencia sobre el carácter no necesario de la articulación de los discursos. Al respecto, la significante obligatoriedad aparece por momentos opuesto a significantes como inclusión educativa o derecho a la educación pese a la articulación densa, por lo menos hasta el 2015, que tuvieron estos significantes por parte del discurso estatal en la esfera nacional. Esto demuestra, una vez más, el carácter no necesario de las articulaciones discursivas, de la precariedad de sutura que estas tienen y de la necesidad de ser constantemente reforzadas a fin de sostener las fijaciones de sentido.

A su vez, resulta preciso diferenciar las políticas en torno a la obligatoriedad de lo político que significa a lo obligatorio. Mientras que la política es el conjunto de prácticas e instituciones a través de las que se crea un determinado orden, lo político es el espacio de poder, conflicto y antagonismo. Es decir, se vincula con los procesos de hegemonización en los que se visibilizan los actos de institución social. Cuando algún elemento de lo político se estabiliza y se vuelve hegemónico se sedimenta en lo social y pasa a formar parte del "orden natural" (Moufee, 2013). Creemos que, si bien el orden selectivo de la escuela secundaria tiende a desestabilizarse, los modos en que se inscribe lo obligatorio suponen un campo abierto de batalla por su significación en donde a las responsabilidades individualizantes se les antepone una perspectiva que inscribe lo obligatorio en el derecho a la escolarización.

Al respecto, en el análisis de los discursos educativos en torno a la escuela secundaria el antagonismo selectividad-obligatoriedad requiere seguir indagándose. Para futuras investigaciones sería relevante analizar las políticas, los discursos educativos y los saberes que los gobiernos de diferente signo político construyeron desde la sanción de la LEN hasta la actualidad en torno a la formación inicial de los/as docentes de escuela secundaria. 
REVISTA DE LA ESCUELA DE CIENCIAS DE LA EdUCACIÓN, AÑo 17, NRO. 16, VOL. 2, JULIO A DiCIEMBRE DE 2021. PÁGINAS 145-153. ISSN 2362-3349 (EN LÍNEA). ESCUELA SECUNDARIA Y FORMACIÓN DOCENTE. SENTIDOS DE LA OBLIGATORIEDAD EN ESTUDIANTES DE PROFESORADO. LUCIANO DE MARCO.

Como mencionábamos al comienzo, la obligatoriedad de la escuela secundaria anuncia el desafío de construir una sensibilidad en el trabajo de enseñar que haga propia la pregunta por la construcción de igualdad. Esto es un desafío que paulatinamente se ha ido procesando en las instituciones que forman docentes de formas disímiles y que requiere futuras indagaciones sobre los modos de traducción que cada institución encuentra para formar a sus estudiantes en esta clave.

Finalmente, y a partir de la información producida en la investigación, resulta significativo problematizar durante la FDI el sentido dominante de la obligatoriedad que desarticula los desafíos políticopedagógicos de las exigencias pedagógico-didácticas. En particular, problematizar los sentidos otorgados a la obligatoriedad a partir de la sanción de la LEN a inicios del siglo XXI en comparación con los otorgados a fines del siglo XIX para el nivel primario. También cabe preguntarse de qué modo se entreteje la política educativa orientada hacia el derecho a la educación con políticas específicamente pedagógicas que provean herramientas y estrategias de enseñanza para la transmisión cultural a las nuevas generaciones, atravesadas por los cambios en las subjetividades que los actuales patrones de sociabilidad forjan y que tienen como centro —en lo que hace a la enseñanza - la variación de los regímenes atencionales. A partir de este estudio y con estas preguntas e interrogantes a cuestas, esperamos realizar, a futuro, nuevos aportes al campo de estudios sobre la formación docente para la escuela secundaria obligatoria del siglo XXI.

\section{Referencias bibliográficas}

Acosta, F. (comp.). (2020). Escolarización y derecho a la educación. En: América Latina en Derecho a la educación y escolarización en América Latina. Buenos Aires: Universidad Nacional de General Sarmiento.

Acosta, F. (2012). La escuela secundaria argentina en perspectiva histórica y comparada modelos institucionales y desgranamiento durante el siglo XX. Cadernos de História da Educaçao, 11, (1), 131-144.

Ball, S. (2015). What is policy? 21 years later: Reflections on the possibilities of policy research. Discourse: Studies in the Cultural Politics of Education,36(3), 306-313.

Birgin, A. (2015). ¿Nuevos docentes, nuevos discursos? Acerca de la expansión de la formación docente en Argentina. En Lopes, A.; Pereira, F; Freitas, M. y De Freitas, A. (Eds.) Trabalho docente, subjetividade eformação, pp. 105-119. Porto, Brasil: Mais Leitura.

Birgin, A. (2017). De la formation des professeurs et de l'élargissement des droits en Argentine. Revue internationale d'éducation de Sèvres. 74, 69-78.

Birgin, A. (2018). Una nueva institución para el gobierno de la formación docente en argentina: el INFD. Revista Práxis Educacional 28 (14), 41-63.

Birgin, A; Moscato, P. e Ingratta, A. (marzo, 2019). ¿Nuevos escenarios en la formación docente? Un análisis desde la normativa y las voces de los y las estudiantes. En II Jornadas de sociología La sociología ante las transformaciones de la sociedad argentina. UNMdP, Mar del Plata.

Capellacci, I. y Miranda, A. (2007). La obligatoriedad de la escuela secundaria. Deudas pendientes y nuevos desafíos. Ministerio de Educación de la Nación, Argentina.

Charovsky, M. (2013). La fragmentación de la formación docente. Su relación con el sistema educativo. Estudio de caso en el partido de Pilar, provincia de Buenos Aires (Tesis de Maestría inédita). Universidad de Buenos Aires, Facultad de Filosofía y Letras. Buenos Aires, Argentina.

Chiroleu, A.; Suasnábar, C. y Rovelli, L. (2012). Política universitaria en la Argentina: revisando viejos legados en busca de nuevos horizontes. Buenos aires, Argentina: Universidad Nacional de General Sarmiento/ Instituto de Estudios y Capacitación (IEC) Federación Nacional de Docentes Universitarios.

Dussel, I. (2004). Inclusión y exclusión en la escuela moderna argentina: una perspectiva postestructuralista. Cadernos de pesquisa. 34(122), 305- 335.

Dussel, I. (2015). Los desafíos de la obligatoriedad de la escuela secundaria. Políticas, instituciones y didácticas en un escenario complejo. En: Tedesco, J. C. (Ed.) La educación argentina hoy. La urgencia del largo plazo. Buenos Aires: Siglo XXI.

Ezcurra, A. (2011). Igualdad en educación superior. Un desafío mundial. Buenos Aires: UNGS.

Falconi, O. (2016). Dispositivos, artefactos y herramientas para el trabajo de enseñar el uso de carpetas, cuadernillos y afiches en el ciclo básico de la escuela secundaria (Tesis de doctorado inédita). Buenos Aires, Argentina: FLACSO.

Giovine R. y Martignoni L. (2011). La escuela media bajo el mandato de la obligatoriedad. Cadernos CEDES, 84, 175 - 194.

Greco, M. B. (2007). La autoridad (pedagógica) en cuestión. Buenos Aires: Homo Sapiens

Krichesky, M. (2013). Estudio sobre la obligatoriedad de la Educación Secundaria en la Provincia de Bs As. Buenos Aires, Argentina: UNIPE.

Laclau, E. y Mouffe, Ch. (1985 [2015]). Hegemonía y estrategia socialista. Hacia una radicalización de la democracia. Buenos Aires: Fondo de Cultura económica.

Larrosa, J. (2019). Esperando no se sabe qué. Sobre el oficio de profesor. C.A.B.A., Argentina: Noveduc

Martignoni, L. (2012). La obligatoriedad de la escuela secundaria. La tensión inclusión - exclusión en las políticas, las instituciones y las experiencias de los actores escolares Espacios en Blanco. Revista de Educación, 22, 7-15. Buenos Aires, Argentina: Universidad Nacional del Centro de la Provincia de Buenos Aires.

Más Rocha, S.; et. al. (Comp.) (2012) La educación secundaria como derecho. Buenos Aires, Argentina: La Crujia.

Masschelein, J. y Simons, M. (2014). Defensa de la escuela. Una cuestión pública. Buenos Aires: Miño y Davila.

Montes, N. y Ziegler, S. (2012). La educación secundaria frente a la obligatoriedad: una ecuación compleja. En Southwell, M. (Ed.), Entre generaciones. Exploraciones sobre educación, cultura e instituciones. Buenos Aires, Argentina: FLACSO Homo Sapiens Editores.

Mouffe, Ch. (2013). En torno a lo político. Buenos Aires: Fondo de Cultura Económica. 


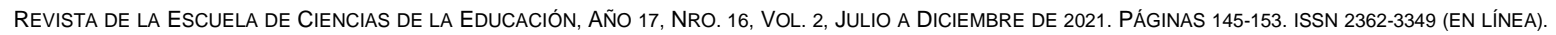
ESCUELA SECUNDARIA Y FORMACIÓN DOCENTE. SENTIDOS DE LA OBLIGATORIEDAD EN ESTUDIANTES DE PROFESORADO. LUCIANO DE MARCO.

Pinkasz, D. (1992). Orígenes del profesorado secundario en la Argentina. En: Braslavsky, C. y Birgin, A. (Ed.), Formación de Profesores. Impacto, pasado y presente. Buenos Aires, Argentina: Miño y Dávila.

Poggi, M. (2018). La educación secundaria una perspectiva sobre América Latina. En Dabenigno, V.; Vinacur, T y Krichesky, M. (Comp.) La educación secundaria en la Ciudad de Buenos Aires: contribuciones del campo de la investigación socioeducativa. Bs. As.: EUDEBA.

Ranciére, J. (1987). El maestro ignorante: cinco lecciones sobre la emancipación intelectual. Buenos Aires, Argentina: Libros del Zorzal.

Sarlo, B. (1998). La máquina cultural. Maestras, traductores y vanguardistas. Buenos Aires, Argentina: Ariel.

Schoo, S. (2013). Redefiniciones normativas y desafíos en la educación secundaria en Argentina. Acuerdos federales en un sistema descentralizado. Serie La educación en Debate (10) Ciudad Autónoma de Buenos Aires, Argentina: Ministerio de Educación de la Nación.

Southwell, M. (2008). Política y educación: ensayos sobre la fijación del significado. En: Cruz Pineda, O. y Echevarría Canto, L. (coord.) Investigación educativa. Herramientas teóricas y análisis político del discurso. Ciudad de México, México: Casa Editorial Juan Pablos.

Southwell, M. (2011). La educación secundaria en Argentina. Notas sobre la historia de un formato. En: Tiramonti, G. (Ed.) Variaciones sobre el formato escolar. Límites y posibilidades de la escuela media. Buenos Aires, Argentina: Homo Sapiens Ediciones, FLACSO.

Southwell, M. (2013). El análisis político del discurso y la cuestión de la politicidad de la educación. En: X Coloquio de Análisis Político del Discurso y Educación. Ciudad de México: DIE-CINVESTAV.

Southwell, M. (2018). El derecho a la educación y la cuestión de lo común. Algunas perspectivas sobre inclusión, universalización y domesticación. Identidades. Núm. 15, Año 8, 61-71

Terigi, F. (2008). Los cambios en el formato de la escuela secundaria argentina: Por qué son necesarios, por qué son tan difíciles. Propuesta Educativa, 29 (1) 63-71.

Vassiliades, A. (2012). Regulaciones del trabajo de enseñar en la provincia de Buenos Aires: posiciones docentes frente a la desigualdad social y educativa. (Tesis de doctorado inédita). Universidad de Buenas Aires, Facultad de Filosofía y Letras, Buenos Aires, Argentina.

Vassiliades, A. (2015). Articulaciones y disputas en la trama entre trabajo docente e igualdad educativa en Argentina. Perfiles Educativos, 37(150), 158-170.

Vassiliades, A. (2020). Impugnaciones de la enseñanza y desplazamientos de la pedagogía: políticas docentes y discursos estandarizados en Argentina durante la presidencia de Mauricio Macri (2015-2019). Espacios en Blanco. Revista de Educación, $\mathrm{N}^{\circ}$ 30, vol. 2, jul./dic. 2020, 247-262. Universidad Nacional del Centro de la Provincia de Buenos Aires, Tandil, Argentina.

Vincent, G.; Lahire, B. y Thin, D. (2001). Sobre a histórica e a teoría da forma escolar. Educacao em Revista. 16, (33).

Williams, R. (1977/2009). Marxismo y literatura. Buenos Aires, Argentina: Las cuarenta. 